\title{
Metastable interstitials in CdSe and CdS crystals
}

\author{
L.V. Borkovska, B.M. Bulakh, L.Yu. Khomenkova, N.O. Korsunska, I.V. Markevich \\ $V$. Lashkaryov Institute of Semiconductor Physics, National Academy of Sciences of Ukraine, \\ 45,pr.Nauky,03028 Kyiv, Ukraine,E-mail:khomen@lumin.semicond.kiev.ua, \\ Fax: +380 (44) 2658342; Phone: +380 (44) 2657234
}

\begin{abstract}
An "anomalous" defect drift in external electric field, namely, transport of acceptorlike centres from the anode to the cathode, has been observed in $\mathrm{CdS}: \mathrm{Cu}, \mathrm{CdS}: \mathrm{Ag}$ and nominally undoped CdSe crystals at 350-700 K. The effect is accounted for by transformation of acceptors into donors under heating. The donors are metastable centres that do not display themselves in the equilibrium state and can be revealed only by drift in electric field. The acceptors are shown to be substitutional impurity atoms, acceptor-to-donor transformation occurring due to transition of these atoms from lattice sites to interstitials. Under cooling reverse donor-to-acceptor transition takes place.
\end{abstract}

Keywords: metastable centres, defect drift in electric field.

Paper received 25.09.03; accepted for publication 11.12.03.

\section{Introduction}

One of the main factors of semiconductor material degradation is transformation of the so-called metastable defects (MDs) under various external influences [1-3]. Such processes also pose a number of fundamental problems essential to physics of defects in semiconductors. That is why MDs are investigated intensively.

It was shown that MDs can be both complex [1,2] and point $[3,4]$ defects. The former rearrange due to their recharge after photocarrier capture and following longrange diffusion of components $[1,2]$, while the later form different centres because of short-range shift occurring in the region of one configuration cage [1,4]. Complex MD's were found earlier in CdS and CdSe crystals and were proved to be responsible for photo-enhanced defect reactions $[1,2]$. The present investigations have shown that, in these compounds, point MD's, namely, metastable interstitials can also be present and display themselves in such phenomenon, as an "anomalous" defect drift under electric field.

Drift of lattice defects under electric field is the wellknown effect. When external electric field is applied to semiconductor at a fit temperature, redistribution of charged mobile defects along the sample occurs, donors being collected near the cathode and acceptors being accumulated near the anode [5]. In $n$-type semiconductor, this redistribution must result in the increase of sample conductivity near the cathode and its decrease near the anode. Such "normal" effect was observed, in particular, in nominally undoped and doped with Li CdS crystals, where mobile shallow donors $\mathrm{Cd}_{\mathrm{i}}$ and $\mathrm{Li}_{\mathrm{i}}$ were shown to be present [6]. Drift of these donors in external electric field caused the increase of conductivity and photosensitivity in the near-cathode crystal region [7]. It has been found, however, that sometimes an "anomalous" effect, namely, the accumulation of acceptors near the cathode can take place. This effect that has been observed in nominally undoped $\mathrm{CdSe}$, as well as in doped with copper and silver CdS crystals, is described and investigated in this work.

\section{Experimental procedure}

Bulk and platelet $\mathrm{CdS}$ crystals doped with $\mathrm{Cu}$ or $\mathrm{Ag}$ and nominally undoped CdSe platelets were investigated. The crystals were highly-resistive: $\rho>10^{5} \mathrm{Ohm} \cdot \mathrm{cm}$ for CdSe and $\rho>10^{9} \mathrm{Ohm} \cdot \mathrm{cm}$ for CdS. All the samples were of ntype, which is usual for CdS and CdSe. Ohmic In or Cd electrodes were applied to the crystals as shown in Fig.1.

In the initial state, i.e. before action of electric field, dark current (DC), as well as photocurrent (PC) and photoluminescence (PL) spectra were measured at 300 or $77 \mathrm{~K}$ between electrodes 1, 2 and 3, 4 (Fig.1, a). Then electrodes 1, 2 and 3, 4 were closed (Fig.1, b), the sample was heated to $T_{d}=350-700 \mathrm{~K}$ and direct electric field $E_{d}=50-100 \mathrm{~V} / \mathrm{cm}$ was applied to it. After a time interval $\Delta t$, the sample was quickly cooled to room temperature, 


\section{L.V. Borkovska et al.: Metastable interstitials in CdSe and CdS crystals ...}

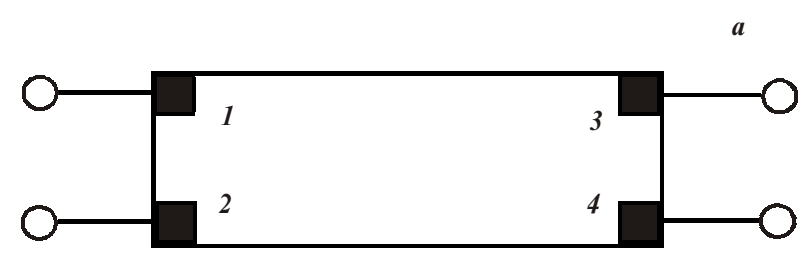

b

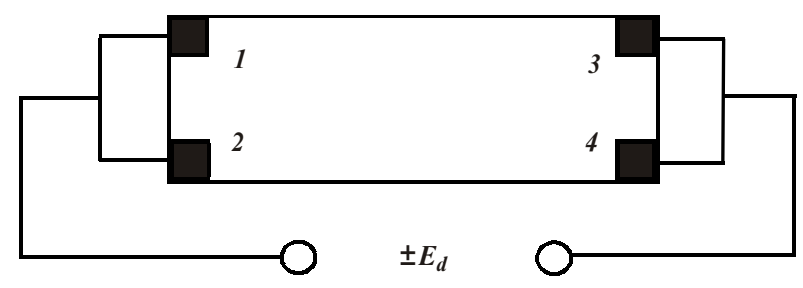

Fig. 1. Appearance of the sample mounted for measurements of DC and PC spectra $(a)$ and for drift carrying out $(b)$.

the electric field was switched off, electrodes 1, 2 and 3, 4 were opened, and above-mentioned characteristics were measured again. To control DC and PC in any sample region, additional indium electrodes were applied between 1,3 and 2,4 ones.

\section{Experimental reasults}

CdSe. The influence of electric field on CdSe crystal characteristics was found to take place already at $T_{d}=350-370 \mathrm{~K}$. After application of $E_{d}=50-100 \mathrm{~V} / \mathrm{cm}$ during $\Delta t=30-40 \mathrm{~s}$ at this temperature, DC and PC values increased at the anode and decreased at the cathode (Fig. 2, a). Simultaneously, the only present in PL spectrum band at $\lambda_{m}=0.93 \mu \mathrm{m}$ strengthened near the anode and quenched near the cathode (Fig. 2, $b$ ). With $\Delta t$ increase, the high-conductivity region spread to the cathode, and at last only thin low-conductivity strip was observed near this electrode, while the rest crystal became highly conductive. If then electric field of opposite direction was applied to the sample at the same $T_{d}$, the lowconductivity region created near the new cathode. This process could be repeated many times, and the results were reproduced.

CdS. In the initial state in PL spectra of CdS:Cu crystals an infrared band at $\lambda_{m}=1.0 \mu \mathrm{m}$ was the most intensive and in PC spectra a strong extrinsic maximum peaked approximately at $0.75 \mu \mathrm{m}$ was present (Fig. 3, $a, b)$.

Application of electric field at $T_{d}=650-700 \mathrm{~K}$ during $\Delta t=3-5 \mathrm{~min}$ resulted in sharp drop of IR-band intensity near the anode and its rise near the cathode, while the intensity of the other PL band did not change noticeably (Fig. 3, a). Simultaneously, PC extrinsic maximum value decreased near the anode and increased near the cathode (Fig. 3, b). The measurements of PL and PC in
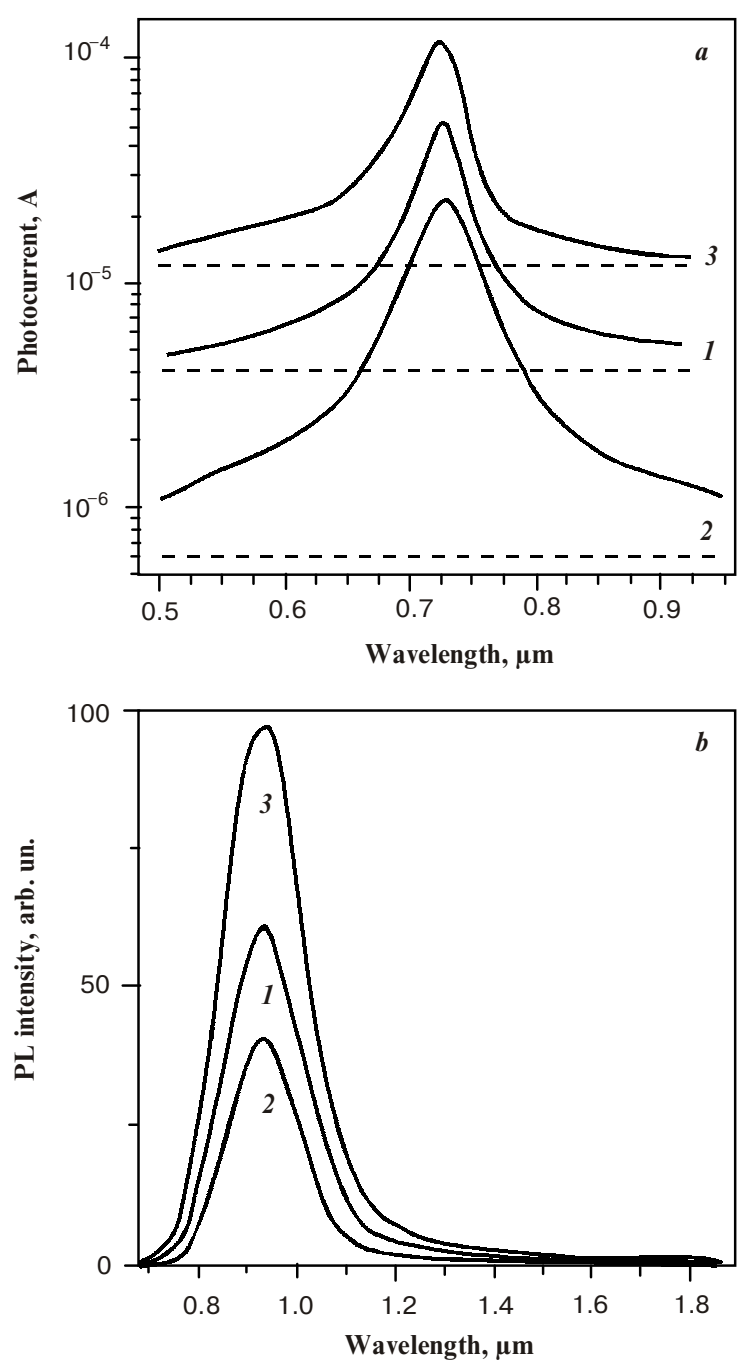

Fig. 2. PC (a) and PL (b) spectra of CdSe crystal at $300 \mathrm{~K}$ (a) and $77 \mathrm{~K}$ (b) measured between the electrodes 1,2 before (1) and after $(2,3)$ the action of electric field, when the electrodes 1,2 were the cathode (2) and the anode (3) $\left(E_{d}=100 \mathrm{~V} / \mathrm{cm}\right.$, $\left.T_{d}=400 \mathrm{~K}, \Delta t_{d}=1 \mathrm{~min}\right)$. The values of dark current are shown as dashed lines.

various sample regions between electrodes 1, 3 and 2, 4 showed that with $\Delta t$ increase the region with quenched IR band and PC extrinsic maximum spread along the sample to the cathode. The effect was reversible: when the sample was heated again and electric field of opposite direction was applied to it, the strengthening of IR band and PC extrinsic maximum near the new cathode and their quenching near the new anode occurred.

Similar changes of PL and PC spectra took place also in CdS:Ag crystals, where in the initial state orange PL band $\lambda_{m}=0.61 \mu \mathrm{m}$ dominated in PL spectra and PC extrinsic maximum peaked at about $0.6 \mu \mathrm{m}$ was observed (Fig. $4 a, b$ ).

The dark currents in investigated $\mathrm{CdS}: \mathrm{Cu}$ and $\mathrm{CdS}: \mathrm{Ag}$ crystals both before and after action of electric field were too small to be measured exactly. 


\section{L.V. Borkovska et al.: Metastable interstitials in CdSe and CdS crystals ...}
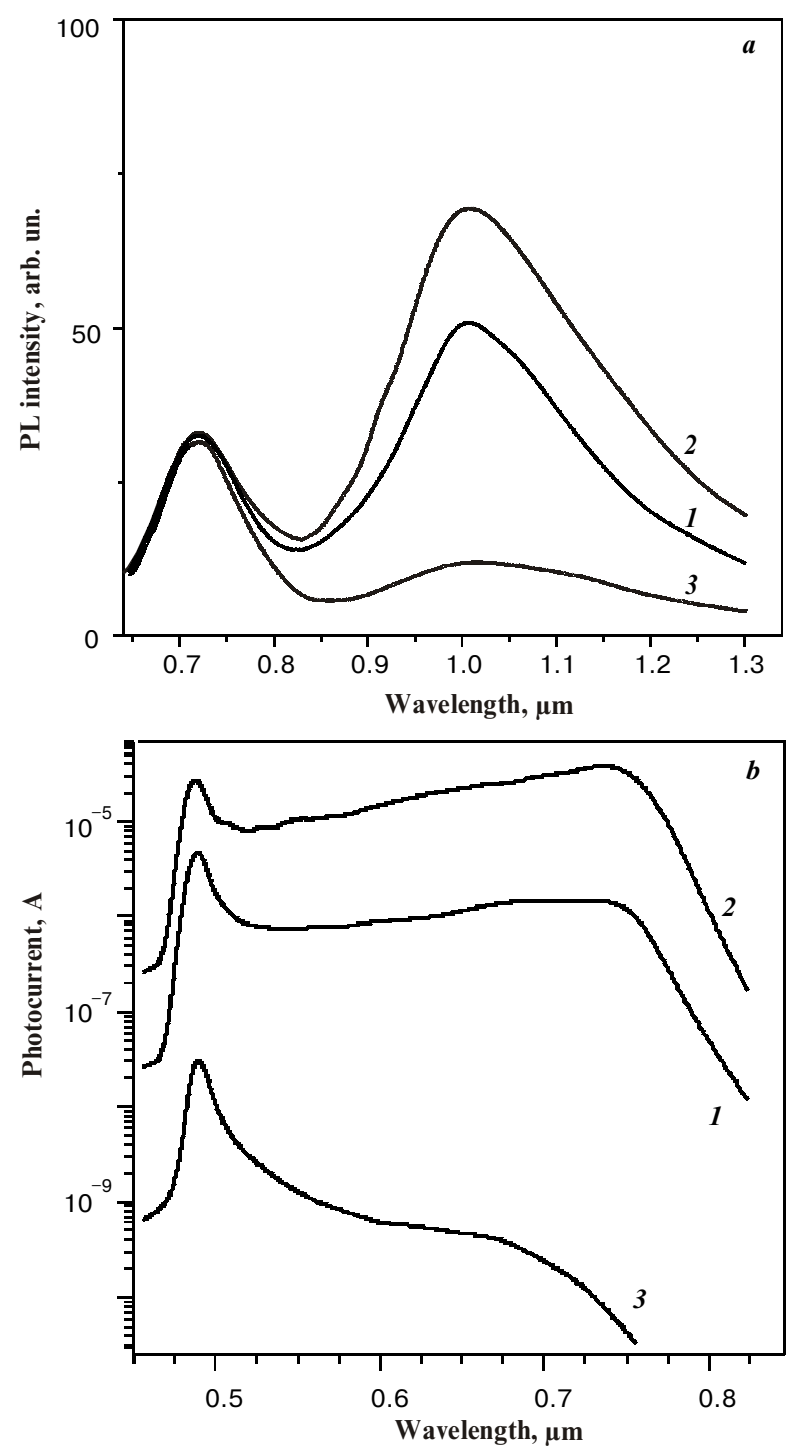

Fig. 3. PL $(a)$ and $\mathrm{PC}(b)$ spectra of $\mathrm{CdS}: \mathrm{Cu}$ crystal at $77 \mathrm{~K}$ measured between electrodes 1,2 before (1) and after $(2,3)$ the action of electric field, when the electrodes 1,2 were the cathode (2) and the anode (3) $\left(E_{d}=70 \mathrm{~V} / \mathrm{cm}, T_{d}=650 \mathrm{~K}, \Delta t_{d}=5 \mathrm{~min}\right.$.)

In the initial state, $\mathrm{CdS}: \mathrm{Cu}$ and $\mathrm{CdS}: \mathrm{Ag}$ crystals had reddish-brown and bright brown colour, respectively. Described above changes in PL and PC spectra were accompanied by the change of crystal colour: after switching electric field on at first, a thin bright yellow (like undoped CdS) strip appeared near the anode. Then, this strip broadened with $\Delta t$ increase, and at last only a thin intensively coloured region was observed near the cathode. Under electric field of opposite direction, the crystal acquired reddish-brown or bright brown colour again, and then bright yellow strip appeared near the new anode.

Induced by electric field characteristic changes kept for many months at $300 \mathrm{~K}$ both for $\mathrm{CdS}$ and CdSe crystals.
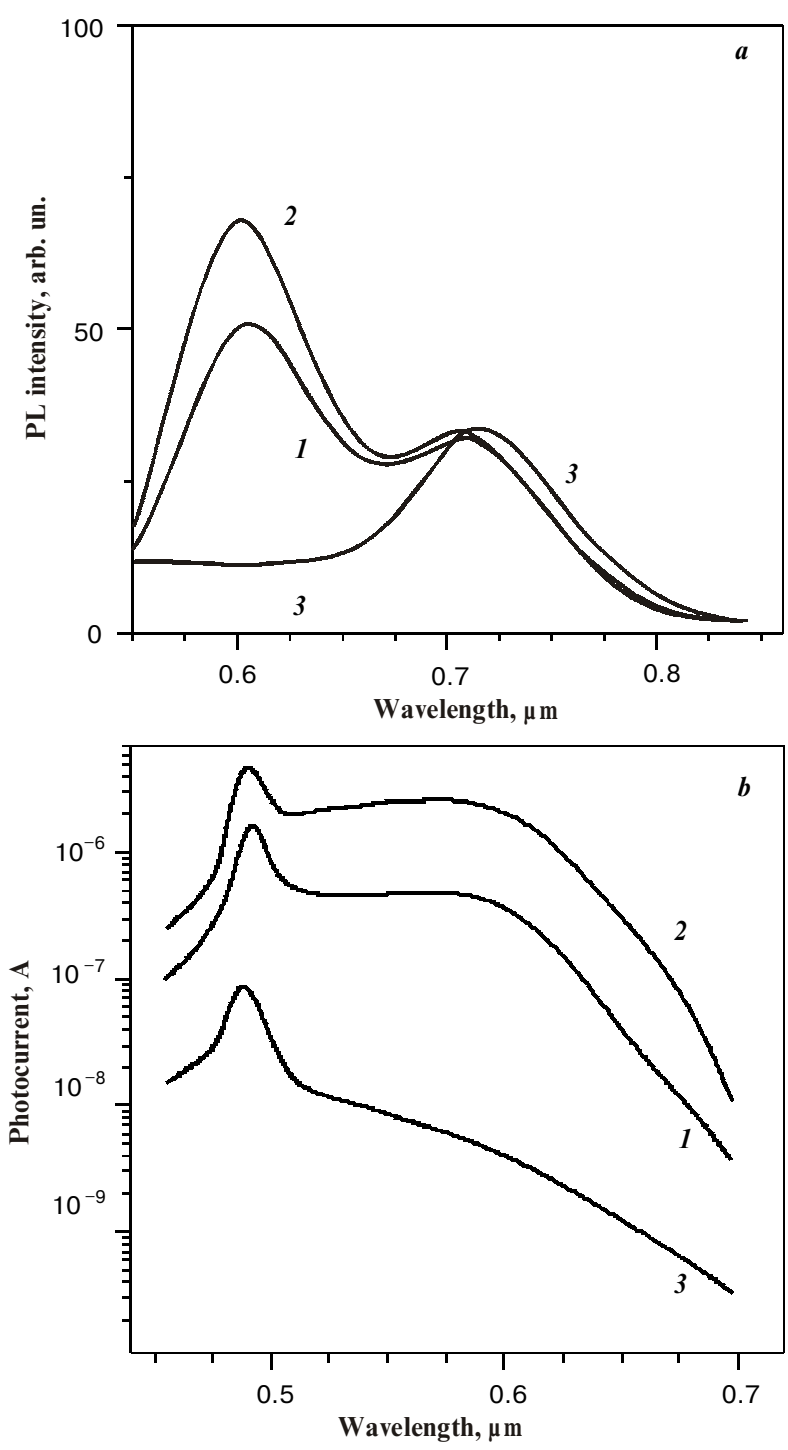

Fig. 4. PL (a) and PC (b) spectra of CdS:Ag crystal at $77 \mathrm{~K}$ measured between the electrodes 1,2 before (1) and after $(2,3)$ action of electric field, when the electrodes 1,2 were the cathode (2) and the anode (3) $\left(E_{d}=90 \mathrm{~V} / \mathrm{cm}, T_{d}=700 \mathrm{~K}, \Delta t_{d}=10 \mathrm{~min}\right)$.

\section{Discussion}

Above results show that, in investigated crystals, transport of acceptors, i.e. negatively charged particles, to the cathode takes place during drift process. Really, redistribution of DC along the sample in CdSe crystals indicates directly that the change of acceptor density with respect to donor one occurs under electric field. The increase of DC in the major part of the sample and its sharp decrease near the cathode testifies that this change is due to extraction of acceptors from the anode-side region and their accumulation at the cathode. This process is not accompanied by the rise of any PL band intensity, so, one can think that the acceptors are nonradiative recom- 


\section{L.V. Borkovska et al.: Metastable interstitials in CdSe and CdS crystals ...}

bination centres. The increase of such centres density must lead to the drop of PC and PL intensity [4], which is observed indeed (Fig. 2, $a, b$ ).

In CdS crystals electric field induces considerable changes in IR (for CdS:Cu) and orange (for CdS:Ag) PL band intensities, while the intensities of other bands remain almost unchanged. This is the evidence that observed PL spectrum transformations result from changes of densities of radiative centres responsible for $\lambda_{m}=$ $=1.0 \mu \mathrm{m}$ and $\lambda_{m}=0.61 \mu \mathrm{m}$ bands [8]. These bands are known to result from recombination of free electrons on $\mathrm{Cu}_{\mathrm{Cd}}$ and $\mathrm{Ag}_{\mathrm{Cd}}$ acceptors respectively, and observed in PC spectra extrinsic maxima were shown to be due to photoionization of electrons from these acceptors to cband $[8,9]$. Since Cucd and Agcd acceptors are «sensitizing» recombination centres $[8,9]$, the values of the intrinsic PC maxima correlate with their densities too. Thus, during drift process, the densities of $\mathrm{Cu}_{\mathrm{Cd}}$ and $\mathrm{Ag}_{\mathrm{Cd}}$ centres decrease in the anode-side region and increase near the cathode.

It is known that $\mathrm{Cu}$ and $\mathrm{Ag}$ incorporate in $\mathrm{CdS}$ and diffuse inside the crystal lattice interstitially as donors $\mathrm{Cu}_{\mathrm{i}}{ }^{+}$and $\mathrm{Ag}_{\mathrm{i}}{ }^{+}$with following reactions $\mathrm{Cu}_{i}+\mathrm{V}_{\mathrm{Cd}} \rightarrow \mathrm{Cu}_{\mathrm{Cd}}$ and $\mathrm{Ag}_{i}+\mathrm{V}_{\mathrm{Cd}} \rightarrow \mathrm{Ag}_{\mathrm{Cd}}$ superimposed on the diffusion processes, diffusivity of interstitials being much more than that of substitutes [10,11]. Acceptors $\mathrm{Cu}_{\mathrm{Cd}}$ and $\mathrm{Ag}_{\mathrm{Cd}}$ are well known radiative recombination centres $[8,12]$, while donors $\mathrm{Cu}_{\mathrm{i}}$ and $\mathrm{Ag}_{\mathrm{i}}$ do not display themselves in the equilibrium state $[10,13]$. Obtained results testify that in previously doped crystals reverse reactions $\mathrm{Cu}_{\mathrm{Cd}} \rightarrow \mathrm{Cu}_{\mathrm{i}}+\mathrm{V}_{\mathrm{Cd}}$ and $\mathrm{Ag}_{\mathrm{Cd}} \rightarrow \mathrm{Ag}_{\mathrm{i}}+\mathrm{V}_{\mathrm{Cd}}$ are intensive enough at $650-700 \mathrm{~K}$. Because of these processes, the impurity atoms acting at $300 \mathrm{~K}$ as acceptors, at elevated temperatures drift in electric field as donors and become acceptors again under cooling. So, the impurities are extracted from the anodeside sample region and accumulated near the cathode. The change of crystal colour after the action of electric field confirms this conclusion.

Transformation process similar to described above takes place, obviously, in CdSe crystals. Although these crystals were nominally undoped, none of native defects could play the role of the centre under consideration. Really, a distinctive feature of drifting defects in CdSe is their high diffusivity. The only native defect that is mobile enough at $350-370 \mathrm{~K}$ is shallow donor $\mathrm{Cd}_{i}[6,7,12]$, but it by no means can transform to acceptor. Thus, defects responsible for anomalous drift in CdSe crystals must be some residual impurity atoms, in all probability, in cadmium sublattice, because $\mathrm{Cd}$ substitutes are much more mobile than Se ones [5,12]. Therefore, elements of the first Group should be considered. The impurity, however, is neither $\mathrm{Cu}$ or $\mathrm{Ag}$, nor Li because: $i$ ) diffusion of $\mathrm{Cu}$ or $\mathrm{Ag}$ in CdSe at $350-370 \mathrm{~K}$ is too slow to explain observed effect [13,14]; ii) $\mathrm{Cu}_{\mathrm{Cd}}$ and $\mathrm{Ag}_{\mathrm{Cd}}$ acceptors in CdSe are radiative centres $[8,9,12]$ and should be observed in PL spectra; iii) although $\mathrm{Li}_{\mathrm{i}}$ has high mobility (its drift is observed already at $250 \mathrm{~K}[6,7]$ ), $\mathrm{Li}_{\mathrm{Cd}} \rightarrow \mathrm{Li}_{i}$ transition was not found in $\mathrm{CdS}$ and CdSe crystals up to $700 \mathrm{~K}$ [13]. Since, in CdSe platelets growth, $\mathrm{H}_{2} \mathrm{Se}$ va- pour was present, one can suppose that the impurity under consideration is hydrogen. This impurity is known to have the highest mobility in semiconductors $[5,12]$ and to form metastable centres in some of them [4].

In conclusion, an "anomalous" defect drift in electric field, namely, transport of acceptors from the anode to the cathode was observed in $\mathrm{CdS}: \mathrm{Cu}, \mathrm{CdS}: \mathrm{Ag}$ and nominally undoped CdSe crystals at 350-700K. The effect was accounted for by transformation of acceptors into donors under heating. Transformation process was concluded to consist in replacement of substitutional impurity atoms from $\mathrm{Cd}$ sites to interstitial positions. In $\mathrm{CdS}$ crystals, impurities responsible for anomalous drift have been shown to be $\mathrm{Cu}$ and $\mathrm{Ag}$. In $\mathrm{CdSe}$ platelets the impurity under consideration is supposed to be hydrogen.

\section{Acknowledgments}

This work was supported by National Academy of Sciences of Ukraine, and one author (L.Yu.K.) were supported by Grant of the President of Ukraine for young scientists.

\section{References}

1. M.K. Sheinkman, L.C. Kimerling, in Defect Control in Semiconductors, ed. K. Sumino, Elsevier Science Publishers B.V. (North-Holland), (1990).

2. M.K. Sheinkman, N.E. Korsunska, I.V. Markevich, T.V. Torchinskaya, The recharge-enhanced transformation of donor-acceptor pairs and clusters in CdS // Phys.Chem.Sol., 43(5), pp. 475-479 (1982).

3. D.V.Lang, Recombination-enhanced reactions in semiconductors // Annu. Rev. Mater. Sci, Paolo Alto, Calif., 12, pp. 377-400, (1982).

4. G.D.Watkins, Metastable defects in silicon: hints for DX and EL2? // Semicond.Sci.Technol., 6(10B), pp. 111-120 (1991).

5. B.J. Boltaks, Diffuziya v poluprovodnikah / Fizmatgiz, Moskva, (1961).

6. N.E. Korsunsaya, I.V. Markevich, T.V. Torchinskaya and M.K. Sheinkman, J.Phys.C: Solid St.Phys. Electrodiffusion of shallow donors in CdS crystals, 13(4), pp. 2975-2978 (1980).

7. N.E. Korsunsaya, I.V. Markevich, I.Yu. Shably and M.K. Sheinkman, Drift of interstitials under electric field in pure and doped with Li CdS crystals // Fiz.Tech.Poluprovod. 15(2), pp.279-282 (1981).

8. V.Ye. Lashkarev, A.V. Lyubchenko and M.K. Sheinkman, Nonequillibrium processes in photoconductors / Naukova Dumka, Kiev, (1981)

9. R.H. Bube, Photoconductivity of solids, Eds.John Willey and Sons, New-York - London, (1960).

10. J.A. Sullivan, Diffusion and solubility of $\mathrm{Cu}$ in $\mathrm{CdS}$ single crystals // Phys. Rev. 184(3), pp. 796-805, (1969).

11. B.L. Timan and Yu. A. Zagoruiko, The charge and mechanism of impurity transfer in CdS crystals Fiz. Tverd. Tela 21(9), pp. 2949-2851, (1979)

12. M. Aven and J.S. Prener (Eds), Physics and chemistry of IIVI compounds // North-Holland publishing company, Amsterdam, (1967).

13. N.E. Korsunska, I.V. Markevich, L.V. Borkovska, L.Yu. Khomenkova, M.K.Sheinkman, O.Yastrubchak, Investigation of lattice defects by means of their drift under electric field // Physica B, 308-310, pp. 967-970 (2001).

14. B.L. Timan and Yu.A. Zagoruiko, Diffusion of $\mathrm{Cu}$ and $\mathrm{Ag}$ in CdSe, Izv. Acad.USSR ser.Neorg. mat. 16(9), pp. 755-756 (1980). 